\title{
ESCRITÓRIOS VIRTUAIS NA WEB: características sobre virtualidade
}

Eliane Maria Stuart Garcez - E-mail: garcez@hu.ufsc.br Gleisy Regina Bóries Fachin - E-mail: gleisy@,ced.ufsc.br ${ }^{1}$ Ada Mariza Tobal-E-mail: tobal@daex.ufsc.br

\section{Resumo}

Análise de sites da Internet denominados escritórios virtuais. Descreve características inerentes às organizações virtuais e identifica empresas que se dizem "virtuais", inserindo-as e comparando-as com os vários conceitos encontrados na literatura, através de método aplicado a oito empresas virtuais. Dessa forma, conseguiu-se comprovar que $50 \%$ das empresas analisadas não passam de empresas convencionais, fazendo uso da Internet.

Palavras-chave: Organizações virtuais; Escritórios virtuais; Critérios de Avaliação.

\begin{abstract}
Analisys of Internet site called virtual office. Description the inherent characteristics to virtual organization, its possiblem to identify those companies know as "virtual", insorting then and companing them with several concepts found in the literature, through of method applied to 8 virtual companies. In this way, it is confirmed that $50 \%$ of the above-mentioned companies are, in fact, conventional companies using the Internet.
\end{abstract}

Keywords: Virtual organization; Virtual office; Evaluation criterian.

\section{INTRODUÇÃO}

Objetiva-se neste artigo, analisar empresas (ditos escritórios) virtuais selecionados aleatoriamente na Revista Guia da Internet, que foram avaliados com o objetivo de comprovar (desmistificar) através desta amostragem, que muitas das empresas que se dizem virtuais não estão de acordo com os conceitos de virtualidade, encontrados na literatura.

Desta forma, foram ressaltadas 13 (treze) características consideradas essenciais, de acordo com os autores Greiner \& Metej (1995), Sieber \& Griese (1995), Martin (1996), sobre o que seriam as organizações virtuais, aplicados aos sites selecionados com o intuito de comprovar sua virtualidade.

Este estudo não pretende ser exaustivo quanto a análise das organizações e escritórios virtuais, pois o campo é vasto e acredita-se que muitas pesquisas estão sendo desenvolvidas e disseminadas nesta área. Acompanhando a crescente evolução das tecnologias associadas as facilidades de comunicação, a cada dia novas informações são divulgadas sobre o uso de escritórios virtuais. A seguir, apresentam-se algumas definições básicas para o entendimento do que é o virtual, do que são escritórios virtuais, convencionais e organizações virtuais. Apresentam-se, igualmente, a metodologia, os critérios e empresas selecionados, os resultados e as conclusões.

\section{CONCEITUAÇÃO}

O termo escritório virtual utilizado aqui não se refere ao "virtual office", que nos Estados Unidos quer dizer teletrabalho.

Segundo Martin (1996, p.115), o termo virtual significa "algo que parece existir, e é usado como se existisse, onde na atualidade não existe".

Para Lévy (1996, p. 16), a palavra virtual é empregada com freqüência para significar "a pura e simples ausência de existência, a realidade, supondo uma efetuação material, uma

\footnotetext{
${ }^{1}$ Alunas do Curso de Pós-Graduação em Engenharia da Produção, da Universidade Federal de Santa Catarina. Disciplina de Introdução à Mídia e Conhecimento, $1^{\circ}$ semestre de 1999.
} 
presença tangível (...). Trata-se de um modo de ser, fecundo e poderoso, que põe em jogo processos de criação, abre futuros, perfura poços de sentido sob a plenitude da presença física imediata (...) é derivado pela transmissão de pacotes através de uma rede."

Escritório virtual para Ferreira (1987), é o "lugar onde se faz o expediente relativo a qualquer administração, se tratam negócios ou se recebem clientes".

Para Zimmerman (1998), a "empresa [convencional] é geralmente associada com um objeto durável limitado, constituído de pessoas e prédios e baseado em uma estrutura de trabalho legal".

Por tratar-se de uma área emergente, destacamos a seguir mais algumas definições de organizações virtuais:

"A organização é uma rede temporária de instituições independentes, empresas ou indivíduos especializados que através do uso da informação e tecnologia da comunicação se unem espontaneamente para utilizar uma vantagem competitiva aparente. Elas se integram verticalmente, trazem seus centros de competências e atuam como se parecessem uma única unidade organizacional (...) que faz substancialmente mais uso de tecnologias da informação e comunicação que presença física para interagir, conduzir negócios e operar conjuntamente, a fim de realizar seus objetivos". (Sieber \& Griese, 1995, p. 9)."

Para a autora, uma organização virtual não pode ser classificada como permanente, pois uma das características básicas da organização virtual é a sua natureza dinâmica.

Em contrapartida, para Martin (1996, p. 117), "existem basicamente dois tipos de virtualidade corporativa. O primeiro relaciona o espaço virtual: empregados na mesma companhia podem estar dispersos geograficamente, mas ligados (linked) como se eles estivessem no mesmo prédio. O segundo se relaciona com negócio virtual: onde os empregados em companhias diferentes podem estar ligados (linked) como se eles fossem da mesma companhia".

De acordo com o artigo da revista PC World (1998) "Os serviços de escritórios virtuais ainda estão longe da maturidade, mas são ferramentas que podem resolver problemas do mundo real hoje. O truque é casar as necessidades que você tem com o produto certo. Nenhum serviço é capaz de fazer tudo e alguns são mais adequados para tarefas específicas". Sendo assim, os componentes mais adequados e necessários para a concepção de um escritório virtual, seriam:

1. Uma página Web para o grupo de trabalho, onde deverão constar links para homepage pessoais e de referências;

2. Uma central de documentos fácil de usar (documents);

3. Configuração e administração simples (administration);

4. Segurança infalível para todas as operações (encryption);

5. Facilidade de arquivamento de dados (shutdown) e arquivamento de dados (signofflarchive);

6. Grupos de discussões que permitam anexar arquivos (discussions);

7. Compatibilidade com browsers de navegação popular, tipos: Internet Explorer, Netscape Navigator 3.0 ou posterior;

8. Compatibilidade embutida com o Microsoft Netmeeting para voz e videoconferência (Microsoft Netmeeting);

9. Apresentar desempenho competitivo quanto ao de quaisquer outros Web sites;

10. Apresentar características estruturais de empresas reais, apesar de ser funções similares na imaginação do observador/cliente.

Considerando aspectos característicos investigados na literatura e as constantes pesquisas na Web, as quais a cada dia se modificam, identifica-se dois pontos fundamentais em se falando de organizações virtuais, segundo Zimmermann (1998):

\section{Visão Institucional}

- Unidades independentes funcionalmente;

- Centros de competências;

- Temporárias; 
- Compartilhamento de recursos e conhecimento;

- Uso de tecnologia da informação;

- Sem competição/propósito comum;

- Sem supervisão, controle baseado em confiança.

\section{Visão funcional}

- Atributo de toda organização;

- Constituição interna e externa;

- Montagem de competências críticas requeridas;

- Requer aprendizagem e orientação adaptativa;

- Capacidade de processamento de informação superior;

- Processos de negócios utilizando-se valor agregado;

- Instituições independentes, empresas ou indivíduos especializados.

Já Goodman, Nagel, Preiss (1995, p. 193), citam 5 características principais de uma organização virtual: oportunismo, excelência, tecnologia, ausência de fronteira e confiança. Enfocam, também, que ela é diferente de outras estruturas organizacionais reais, principalmente para tirar proveito de oportunidades e ser desativada assim que o objetivo for alcançado. Uma organização virtual deve ter padrão mundial e excelência em termos de competências, deve oferecer tecnologia de padrão mundial em suas soluções de produtos e serviços. Para finalizar, os autores reforçam a tese das competências complementares e fisicamente distribuídas, permanecendo dispersas e não obstante transformarem-se numa fonte produtiva consistente, cuja natureza é invisível e sem fronteiras do ponto de vista dos clientes.

Segundo Franke (apud Sieber \& Griese, 1995, p. 17), as principais diferenças entre organizações virtuais e outras formas de cooperação são:

- Contribuição de centros de competências;

- Independência empresarial;

- Alta flexibilidade;

- Parcerias unidas rapidamente, sem longas negociações;

- Parcerias desfeitas sem qualquer problema;

- As companhias podem ser parceiras de várias corporações virtuais.

\section{METODOLOGIA}

Para este trabalho foram selecionadas oito empresas no Guia da Internet, as quais possuíam maior números de informações em seus sites e de áreas distintas na prestação de serviços e identificavam-se como escritórios virtuais.

Considerando as colocações dos autores Greiner \& Metej (1995), Sieber \& Griese (1995), Martin (1996), foram identificados alguns critérios de avaliação, considerados como essenciais para uma empresa virtual, com os quais foram analisados os oito sites, aplicando-se os critérios de avaliação, apresentados a seguir.

\subsection{Critérios para avaliação:}

1. Competências básicas (time de estrelas, eles se formam para criar o melhor de tudo);

2. Valor agregado (quando agrega conhecimento, ou uma idéia);

3. Supervisão/controle, baseado em confiança; 
4. Sem competição, propósito comum; está corretíssima

5. Temporárias (poderão ser desativadas assim que os objetivos forem alcançados);

6. Independentes (elas podem participar de outras redes);

7. Uso de tecnologia da informação;

8. Se integram verticalmente (não existe uma estrutura hierárquica);

9. Dinâmicas;

10. Compartilhamento de recursos (financeiros, tecnológicos e conhecimento);

11. Tempo independente, acredito que esta palavra independente não é necessária, somente tempo ou podes usar direto horário flexível, o problema é que o autor usa somente tempo(horário flexível, fusos horários);

12. Sem Espaço físico (não possui localização física);

13. Teia virtual (é um conjunto de parceiros pré-qualificados que concordam em formar um grupo de membros potenciais de organizações virtuais).

\subsection{Empresas virtuais estudadas:}

1. SEBRAE: Serviço de Apoio às Micros e Pequenas Empresas - organização que colocou um serviço à disposição de seus clientes: trata-se de um escritório virtual para locações temporárias, com: sala de reuniões, serviços, acessórios e material de apoio, um microcomputador com softwares instalados e acesso à Internet. Localiza-se no World Trade Center de São Paulo, telefone 0800-78020 (Brasil) ou no URL: http://www.listasamarelas.com.br/oesp/revista/rpm21697/sebrae.html. Em 06/06/1999, testando o endereço, constatou sua inclusão no site do SEBRAE - http://www.sebrae.com

2. CIEE: Centro de Integração Empresa Escola - órgão que coordena um trabalho de contato entre estudantes, escolas e empresas. Instituição de direito privado, que ajuda os estudantes a aprimorarem sua qualificação profissional através de concessão de estágios remunerados nas empresas conveniadas. Localiza-se em São Paulo, telefone 0118299787 (Brasil), e possui filiais em todos os estados do Brasil ou na URL: http://www.ciee.org.br/imp.html - conferido em 06/06/1999 e localizado no endereço URL: http://www.ciee.org.br/

3. CATHO: Recolocação virtual de executivos e profissionais especializados - empresa que presta assessoria de consultores especializados, através de métodos e tecnologias avançadas, para pessoas cadastradas que buscam novas oportunidades de trabalho em qualquer região do Brasil, através da Internet. aarruda@catho.com.br ou na URL: http://www.catho.com.br/virtual.html

4. BUSINESS CENTER - VIRTUAL OFFICE - diz ser a primeira empresa virtual no estado de Santa Catarina e pioneira em locação de escritórios. Espaço idealizado para suprir em tempo integral ou parcial, necessidades dos clientes (empresas convencionais, virtuais, teletrabalhadores e profissionais liberais). As tecnologias disponíveis são: videocassete, televisão, computadores, notebooks, transcoders e Internet. Localizada em Florianópolis/SC (Brasil). http://www.bcenter.com.br

5. SMART OFFICE: Consultoria e Serviços Ltda - organização que oferece serviços a clientes através da Internet, tais como: instalação, desenvolvimento de Websites, instalação de redes Windows, automação de documentos, desenvolvimento e manutenção de sistemas, na URL: http://www.task.com.br/smartoffice/services.htm

6. SITEBR: Serviços de Colocação de Site na Internet - empresa virtual que tem o papel de consultoria, produção e prestação de serviços de Internet. Providenciam tudo, criando, implantando e mantendo sites para clientes, e oferecendo consultoria operacional e técnica, no endereço: Webmaster@sitebr.com ou na URL: http://www.sitebr.com/index.html 
7. THE VIRTUAL TUTOR'S HOME PAGE: Curso de Inglês via Correio Eletrônico empresa com uma equipe de professores que prepara, envia e corrige exercícios da língua inglesa, via correio eletrônico. Localização em São Paulo, telefone/fax 551150719773 (Brasil), endereço: virtualtutor@ilm.net e ucarval@ilm.net e URL: http://home.ican.ntl/ 712655

8. VIRTUAL OFFICE - apresenta-se como uma estrutura completa de serviços e representações, para empresas e profissionais liberais, dispondo de ferramentas administrativas e logísticas de alto nível. Oferecem também assessoria de marketing, abertura de empresas, assessoria contábil completa (análise tributária), logomarcas, comunicação visual, etc.... Localiza-se em São Paulo, no telefone 01172958533 (Brasil), e no virtualoffice@alphanet.com.br e URL: http://www.virtualoffice.com.br/html

A partir dos critérios foram analisadas as oito empresas, comparando-as com os 13 critérios. A seguir, montou-se um quadro para verificar se as empresas citadas poderiam ser consideradas virtuais. Os resultados podem ser visualizados no quadro 1.1.

Foi utilizado para identificar cada critério positivo a letra S (SIM) e N (NÃO) quanto o critério era negativo. Sendo que ao final, cada empresa totaliza os critérios positivos, apresentados em porcentagem (\%).

Foram consideradas como escritórios virtuais as empresas que preencheram todos os critérios de forma positiva, portanto totalizando 13 critérios positivos $=100 \%$.

\section{QUADRO 1 - CRITÉRIOS PARA MEDIR UMA EMPRESA VIRTUAL}

\begin{tabular}{|c|c|c|c|c|c|c|c|c|}
\hline$\widehat{C r i t e r i e s ~}^{\text {Empresas }}$ & SEBRAE & CIEE & CATHO & $\begin{array}{c}\text { BUSINESS } \\
\text { CENTER }\end{array}$ & $\begin{array}{l}\text { SMARTO } \\
\text { FFICE }\end{array}$ & SITEBR & $\begin{array}{c}\text { THE } \\
\text { VIRTUAL } \\
\text { TUTOR's }\end{array}$ & $\begin{array}{l}\text { VIRTUAL } \\
\text { OFFICE }\end{array}$ \\
\hline $\begin{array}{c}\text { Competências } \\
\text { básicas }\end{array}$ & $\mathrm{S}$ & $\mathrm{S}$ & $\mathrm{S}$ & $\mathrm{N}$ & $\mathrm{S}$ & $\mathrm{S}$ & $\mathrm{S}$ & $\mathrm{N}$ \\
\hline $\begin{array}{c}\text { Valor } \\
\text { agregado }\end{array}$ & $\mathrm{S}$ & $\mathrm{S}$ & $\mathrm{S}$ & $\mathrm{N}$ & $S$ & $\mathrm{~S}$ & $\mathrm{~S}$ & $\mathrm{~N}$ \\
\hline $\begin{array}{c}\text { Supervisão/ } \\
\text { Controle }\end{array}$ & $\mathrm{N}$ & $\mathrm{N}$ & $\mathrm{S}$ & $\mathrm{N}$ & $\mathrm{S}$ & $\mathrm{S}$ & $\mathrm{S}$ & $\mathrm{N}$ \\
\hline $\begin{array}{c}\text { Sem } \\
\text { competição, } \\
\text { propósito } \\
\text { comum } \\
\end{array}$ & $\mathrm{N}$ & $\mathrm{N}$ & $\mathrm{S}$ & $\mathrm{N}$ & $\mathrm{S}$ & $\mathrm{S}$ & $\mathrm{S}$ & $\mathrm{N}$ \\
\hline Temporárias & $\mathrm{S}$ & $\mathrm{S}$ & $\mathrm{S}$ & $\mathrm{S}$ & $\mathrm{S}$ & $\mathrm{S}$ & $\mathrm{S}$ & $\mathrm{S}$ \\
\hline Independentes & $\mathrm{S}$ & $\mathrm{S}$ & $\mathrm{S}$ & $\mathrm{S}$ & $\mathrm{S}$ & $\mathrm{S}$ & $\mathrm{S}$ & $\mathrm{S}$ \\
\hline $\begin{array}{c}\text { Uso de } \\
\text { tecnologia da } \\
\text { informação }\end{array}$ & $\mathrm{S}$ & $\mathrm{S}$ & $\mathrm{S}$ & $\mathrm{S}$ & $\mathrm{S}$ & $\mathrm{S}$ & $\mathrm{S}$ & $\mathrm{S}$ \\
\hline $\begin{array}{l}\text { Se integram } \\
\text { verticalmente }\end{array}$ & $\mathrm{N}$ & $\mathrm{N}$ & $\mathrm{S}$ & $\mathrm{N}$ & $\mathrm{S}$ & $\mathrm{S}$ & $\mathrm{S}$ & $\mathrm{N}$ \\
\hline Dinâmicas & $\mathrm{S}$ & $\mathrm{S}$ & $\mathrm{S}$ & $\mathrm{S}$ & $\mathrm{S}$ & $\mathrm{S}$ & $\mathrm{S}$ & $\mathrm{S}$ \\
\hline $\begin{array}{l}\text { Compartilha- } \\
\text { mento de } \\
\text { recursos }\end{array}$ & $\mathrm{S}$ & $\mathrm{S}$ & $\mathrm{S}$ & $\mathrm{N}$ & $\mathrm{S}$ & $\mathrm{S}$ & $S$ & $\mathrm{~N}$ \\
\hline $\begin{array}{c}\text { Tempo } \\
\text { independente }\end{array}$ & $\mathrm{S}$ & $\mathrm{S}$ & $\mathrm{S}$ & $\mathrm{S}$ & $\mathrm{S}$ & $S$ & $\mathrm{~S}$ & $\mathrm{~S}$ \\
\hline $\begin{array}{c}\text { Sem Espaço } \\
\text { físico } \\
\end{array}$ & $\mathrm{S}$ & $\mathrm{S}$ & $\mathrm{S}$ & $\mathrm{S}$ & $\mathrm{S}$ & $\mathrm{S}$ & $\mathrm{S}$ & $\mathrm{S}$ \\
\hline Teia virtual & $\mathrm{N}$ & $\mathrm{N}$ & $\mathrm{S}$ & $\mathrm{N}$ & $\mathrm{S}$ & $\mathrm{S}$ & $\mathrm{S}$ & $\mathrm{N}$ \\
\hline TOTAL & $\begin{array}{c}9 \\
(69,23 \%)\end{array}$ & $\begin{array}{c}9 \\
(69,23 \%) \\
\end{array}$ & $\begin{array}{c}13 \\
(100 \%) \\
\end{array}$ & $\begin{array}{c}6 \\
(46,15 \%) \\
\end{array}$ & $\begin{array}{c}13 \\
(100 \%) \\
\end{array}$ & $\begin{array}{c}13 \\
(100 \%) \\
\end{array}$ & $\begin{array}{c}13 \\
(100 \%) \\
\end{array}$ & $\begin{array}{c}6 \\
(46,15 \%) \\
\end{array}$ \\
\hline
\end{tabular}

Enc. Bibli: R. Eletr. Bibliotecon. Ci. Inf., ISSN 1518-2924, Florianópolis, Brasil, n.8, p.28-34, 1999.32 


\section{RESULTADOS}

Conforme se observa no quadro acima, os resultados foram obtidos através da análise de cada site das empresas selecionadas, comparando-as com os critérios pré-definidos anteriormente. Destaca-se que, dos 13 critérios compilados, estudados e aplicados aos escritórios virtuais, foram obtidos os seguintes resultados.

As empresas CATHO, Smart Office, SiteBr e Virtual Tutor's apresentam 100\% de enquadramento podendo ser consideradas portanto, escritórios virtuais.

O SEBRAE e o CIEE apresentam índices de 69,23\%. São sites considerados dentro da linha de virtual, mas precisam adaptar-se em alguns critérios. As empresas Business Center e a Virtual Office atingiram $46,15 \%$ de critérios positivos sendo, a princípio, consideradas empresas que utilizam a Web como ferramenta para seus negócios, podendo ser consideradas como empresas convencionais.

Segundo Goodman, Nagel, Preiss (1995, p. 192), "é um modelo organizacional que utiliza tecnologia para unir, de forma dinâmica, pessoas, bens e idéias. Ressalta que, embora a tecnologia seja um mecanismo facilitador importante, a longo prazo não é um requisito essencial. A empresa virtual deve contar com a maior adaptabilidade possível, precisa ser dinâmica em sua estrutura organizacional interna, suas normas, regulamentos e assim por diante. Uma organização virtual consiste na integração de principais competências distribuídas entre diversas organizações reais que é definida e impulsionada por oportunidades".

Alguns autores não compartilham com estas idéias. Segundo Hill (apud Sieber, 1995, p. 11), "as organizações virtuais podem ser tão estruturadas quanto as organizações reais". Continuando, levanta-se a seguinte questão: se entre nós existem acordos cooperativos, estáveis e mesmo contratuais, diferentes de empresas independentes, que dependem principalmente de tecnologias de informação e de comunicações para suas operações, isto significa que não há uma organização virtual. O autor afirma que as organizações virtuais pode ser formais/informais, estanque/soltas, hierárquicas/planejadas. Todos estes atributos podem ser características de organizações permanentes, tão bem quanto de organizações temporárias. $\mathrm{Na}$ realidade, as organizações virtuais podem ser tão estruturadas quanto a organizações reais (Idem, p. 23)

Neste aspecto, Goodman, Nagel, Preiss (1995, p. 211) comentam o papel das empresas gigantes na atual tendência de subdivisões das grandes corporações em conjuntos menores de corporações, cada qual responsável por atingir metas e objetivos definidos pela empresa-mãe. De um modo bastante realista, a empresa-mãe ou a grande corporação nada mais é que uma teia de organizações. De fato, a IBM e outras corporações, como a FIAT, a DuPont, a Rubbermaid, e tantas outras, não estão utilizando o conceito de organização virtual só interna, mas externamente. Se for pensada esta teia como um grupo de organizações pré-qualificadas, com uma cultura organizacional compartilhada, e uma ou mais áreas de excelência em termos de oportunidades, talvez se tenha definido o futuro papel das grandes corporações, declaradamente como o de teias.

\section{CONSIDERAÇÕES FINAIS}

A grande dificuldade encontrada nas análises envolvendo a Web está na definição de critérios de avaliação, principalmente se levado em consideração que trata-se de uma área emergente, onde nem tudo esta concretizado, definido, documentado. Portanto, os critérios apresentados no quadro 1, resgatados na literatura, foram utilizados para analisar as empresas selecionadas que poderiam ser consideradas como escritórios virtuais. 
Cabe ressaltar que este trabalho baseou-se na literatura encontrada na área e na relação de empresas apresentadas no Guia da Internet. Não foram efetuados contatos diretos com as empresas envolvidas, pois o objetivo do presente trabalho era demonstrar que necessitamos definir critérios de avaliação técnicos-científicos, a fim de melhor disponibilizar as informações de forma confiável.

Ao analisar os resultados verificamos que, se houver uma avaliação apurada de cada site existente na Web, constatar-se-á que a maioria deles continuam, de uma forma ou outra, estruturados em moldes tradicionais, pois não é fácil quebrar paradigmas, principalmente pela grande flexibilidade e liberdade de expressão na Web, onde encontra-se grande variedade de sites, nos mais diversos formatos e conteúdos.

O Ciberespaço é utilizado pela maioria das empresas como ferramenta para unir competências, disseminar seus produtos e serviços, realizar negócios e principalmente manter-se no mercado, pois é uma questão de sobrevivência no mundo globalizado.

Cabe, entretanto, uma reflexão quanto aos vários sites disponibilizados na Web, sejam eles, comerciais, científicos, particulares, em qualquer linha de atuação. Hoje encontramos todos os tipos mas, quais destes são os de real credibilidade? Quais são os sites que atendem as perspectivas que norteiam a disseminação de informações de cunho legal? Ou será que a Web é a grande teia mãe, onde tudo é permitido?!

\section{REFERÊNCIAS BIBLIOGRÁFICAS}

ESCRITÓRIO virtual. PCWORLD, p.3-10, jun. 1998.

ESCRITÓRIO virtual é a opção contra a correria do dia-a-dia. Diário catarinense. 6 jun. 1999. p. 32

FERREIRA, A. B. de H. Novo dicionário Aurélio da língua portuguesa. 2. ed. Rio de Janeiro : Nova Fronteira, 1999.

GOODMAN, Steve. L., NAGEL, Roger. N., PREISS, Kenneth. Organizações virtuais. São Paulo : Érica, 1995. Cap. 6. : Agile competitors. p. 189-221.

GREINER, Ray \& METEJ, George. Going Virtual: moving your organization into the 21 st Century. New Jersey : Prentice Hall, 1995.

KUGELMASS, Joel. Teletrabalho: novas oportunidades para o trabalho flexível. São Paulo : Atlas, 1996.

LÉVY, P. O que é o virtual? São Paulo : Ed. 34, 1996.

LÉVY, P. Cibercultura. Trad. Carlos Irineu da Costa. São Paulo: Ed. 34, 1999. 260 p. (Coleção TRANS)

MARTIN, James. Cybercorp : the new business revolution. New York : Amacon, 1996. Cap. 6, p. 115-125. : Virtualiness.

Rev. Guia Internet, 18 dez. 1998. (não paginado)

SIEBER, P. \& GRIESE, J. (Eds). Organizational virtualness. In: Proceedings of the VoNetWorkshop, Bern: Apr, 27-28. Resuméé of VoTalk... Bern : Simona Verlag Bern, 1995.

ZIMMERMANN, Frank-O. Structural and managerial aspects of virtual enterprises. http://www.teco.uni-karlsruhe.de/IT-VISION/vu-e-teco/htm 